\title{
On Minimal Generating Sets of Certain Subsemigroups of Isometries
}

\author{
Leyla Bugay* and Melek Yağc1
}

\begin{abstract}
Let $D P_{n}$ and $O D P_{n}$ be the semigroups of all isometries and of all order-preserving isometries on $X_{n}$, respectively. In this paper we investigate the structure of minimal generating sets of the subsemigroup $D P_{n, r}=\left\{\alpha \in D P_{n}:|\operatorname{im}(\alpha)| \leq r\right\}$ (similarly of the subsemigroup $O D P_{n, r}=\left\{\alpha \in O D P_{n}:|\operatorname{im}(\alpha)| \leq r\right\}$ ) for $2 \leq r \leq n-1$.
\end{abstract}

Keywords: Isometry; order-preserving /order-reversing map; (minimal) generating set.

AMS Subject Classification (2020): 20M20.

${ }^{*}$ Corresponding author

\section{Introduction}

Let $I_{n}$ be the symmetric inverse semigroup on the finite chain $X_{n}=\{1, \ldots, n\}$, and let $\alpha \in I_{n}$. If $(\forall x \in \operatorname{dom}(\alpha))$ $x \alpha=x$ then $\alpha$ is called the partial identity map on $U=\operatorname{dom}(\alpha) \subseteq X_{n}$, denoted by id ${ }_{U}$. If $(\forall x, y \in \operatorname{dom}(\alpha))$ $x \leq y \Rightarrow x \alpha \leq y \alpha(x \leq y \Rightarrow x \alpha \geq y \alpha)$ then $\alpha$ is called an order-preserving map (an order-reversing map), and if $(\forall x, y \in \operatorname{dom}(\alpha))|x-y|=|x \alpha-y \alpha|$ then $\alpha$ is called an isometry (or distance-preserving map) on $X_{n}$, under its natural order. Then the subset of all isometries and the subset of all order-preserving isometries, denoted by $D P_{n}$ and $O D P_{n}$ respectively, that is,

$$
\begin{aligned}
D P_{n} & =\left\{\alpha \in I_{n}:(\forall x, y \in \operatorname{dom}(\alpha)) \quad|x-y|=|x \alpha-y \alpha|\right\} \text { and } \\
O D P_{n} & =\left\{\alpha \in D P_{n}:(\forall x, y \in \operatorname{dom}(\alpha)) \quad x \leq y \Rightarrow x \alpha \leq y \alpha\right\}
\end{aligned}
$$

are clearly subsemigroups of $I_{n}$ and $O D P_{n} \subseteq D P_{n} \subseteq I_{n}$. Moreover, for $2 \leq r \leq n-1$, let

$$
\begin{aligned}
D P_{n, r} & =\left\{\alpha \in D P_{n}:|\operatorname{im}(\alpha)| \leq r\right\} \text { and } \\
O D P_{n, r} & =\left\{\alpha \in O D P_{n}:|\operatorname{im}(\alpha)| \leq r\right\}
\end{aligned}
$$

which are clearly subsemigroups of $D P_{n}$ and $O D P_{n}$, respectively.

Let $S$ be any semigroup, and let $W$ be any non-empty subset of $S$. Then the subsemigroup generated by $W$, that is, the smallest subsemigroup of $S$ containing $W$, is denoted by $\langle W\rangle$. The rank of a finitely generated semigroup $S$, i.e., a semigroup generated by a finite subset, is defined by

$$
\operatorname{rank}(S)=\min \{|W|:\langle W\rangle=S\}
$$

Moreover, the generating set of $S$ with cardinality $\operatorname{rank}(S)$ is called a minimal generating set of $S$.

Al-Kharousi, Kehinde and Umar showed in [1, Theorems 3.1, 3.4 and 3.5] that

$$
\begin{aligned}
& \operatorname{rank}\left(O D P_{n, n-1}\right)=n, \quad \operatorname{rank}\left(O D P_{n}\right)=n+1, \\
& \operatorname{rank}\left(D P_{n, n-1}\right)=n, \text { and } \operatorname{rank}\left(D P_{n}\right)=\left\lfloor\frac{n+3}{2}\right\rfloor .
\end{aligned}
$$

Received : 19-04-2020, Accepted : 22-06-2020 
Then, in [2], we introduced some properties of $D P_{n, r}$ and $O D P_{n, r}$, and also showed that

$$
\operatorname{rank}\left(D P_{n, r}\right)=\left(\begin{array}{l}
n \\
r
\end{array}\right) \text { and } \operatorname{rank}\left(O D P_{n, r}\right)=\left(\begin{array}{l}
n \\
r
\end{array}\right) .
$$

However, there were no results about the structure of any minimal generating set of $D P_{n, r}\left(O D P_{n, r}\right)$ and no method for whether an arbitrary non-empty subset $X$ of $D P_{n, r}\left(O D P_{n, r}\right)$ is a minimal generating set of $D P_{n, r}$ $\left(O D P_{n, r}\right)$, or not for $2 \leq r \leq n-1$. Thereby, in this study we improve a useful method to respond this lack.

\section{Preliminaries}

In this section we remind some definitions and properties given also in [2], and without otherwise stated we take $2 \leq r \leq n-1$.

Let $\alpha \in D P_{n}$, and let dom $(\alpha)=\left\{a_{1}<\cdots<a_{p}\right\}$ with $2 \leq p \leq n$. Then the gap and the reverse-gap of $\alpha$, denoted by $\mathrm{g}(\alpha)$ and $\mathrm{g}^{R}(\alpha)$, are defined by

$$
\mathrm{g}(\alpha)=\left(d_{1}, \ldots, d_{p-1}\right) \text { and } \mathrm{g}^{R}(\alpha)=\left(d_{p-1}, \ldots, d_{1}\right),
$$

respectively, where $d_{i}=a_{(i+1)}-a_{i}$ for each $1 \leq i \leq p-1$. It is easy to see that $p-1 \leq \sum_{i=1}^{p-1} d_{i} \leq n-1$ for any gap $\left(d_{1}, \ldots, d_{p-1}\right)$. Moreover, for any ordered $(p-1)$-tuple $\left(d_{1}, \ldots, d_{p-1}\right)$, if

$$
\left(d_{1}, \ldots, d_{p-1}\right)=\left(d_{p-1}, \ldots, d_{1}\right),
$$

then $\left(d_{1}, \ldots, d_{p-1}\right)$ is called symmetric (otherwise, asymmetric) ordered ( $\left.p-1\right)$-tuple.

From [1, Lemma 1.2] we know that each element of $D P_{n}$ is either order-preserving or order-reversing map. Let $\alpha \in D P_{n}$ such that $\operatorname{dom}(\alpha)=A=\left\{a_{1}<\cdots<a_{p}\right\}$ and $\operatorname{im}(\alpha)=B=\left\{b_{1}<\cdots<b_{p}\right\}$ for $2 \leq p \leq n$. If $\alpha$ is an order-preserving map, then $a_{i+1}-a_{i}=b_{i+1}-b_{i}$ for each $1 \leq i \leq p-1 \leq n-1$ and $\alpha$ has the following tabular form:

$$
\alpha=\left(\begin{array}{cccc}
a_{1} & a_{2} & \cdots & a_{p} \\
b_{1} & b_{2} & \cdots & b_{p}
\end{array}\right), \text { or shortly } \alpha=\left(\begin{array}{c}
A \\
B
\end{array}\right) .
$$

If $\alpha \in D P_{n}$ is an order-reversing map, then $a_{i+1}-a_{i}=b_{p-i+1}-b_{p-i}$ for each $1 \leq i \leq p-1 \leq n-1$ and $\alpha$ has the following tabular form:

$$
\alpha=\left(\begin{array}{cccc}
a_{1} & a_{2} & \cdots & a_{p} \\
b_{p} & b_{p-1} & \cdots & b_{1}
\end{array}\right), \text { or shortly } \alpha=\left(\begin{array}{c}
A \\
B^{R}
\end{array}\right) .
$$

From the definitions of the Green's equivalences we clearly have

(i) $\alpha \mathcal{R} \beta \Leftrightarrow \operatorname{dom}(\alpha)=\operatorname{dom}(\beta)$,

(ii) $\alpha \mathcal{L} \beta \Leftrightarrow \operatorname{im}(\alpha)=\operatorname{im}(\beta)$ and

(iii) $\alpha \mathcal{H} \beta \Leftrightarrow \operatorname{dom}(\alpha)=\operatorname{dom}(\beta)$ and $\operatorname{im}(\alpha)=\operatorname{im}(\beta)$

for $\alpha, \beta \in D P_{n, r}$ or $\alpha, \beta \in O D P_{n, r}$, and we have

(iv) $\alpha \mathcal{D} \beta \Leftrightarrow \mathrm{g}(\alpha)=\mathrm{g}(\beta)$ or $\mathrm{g}(\alpha)=\mathrm{g}^{R}(\beta)$ for $\alpha, \beta \in D P_{n, r}$ and

(v) $\alpha \mathcal{D} \beta \Leftrightarrow \mathrm{g}(\alpha)=\mathrm{g}(\beta)$ for $\alpha, \beta \in O D P_{n, r}$.

(For the definitions of Green's equivalences and for the other terms in semigroup theory, which are not explained here, we refer to $[3,4])$.

Let $K_{p}=\left\{\alpha \in D P_{n}:|\operatorname{im}(\alpha)|=p\right\}$ and let $L_{p}=\left\{\alpha \in O D P_{n}:|\operatorname{im}(\alpha)|=p\right\}$ for $0 \leq p \leq n$. Then $K_{p}\left(L_{p}\right)$ is disjoint union of some $\mathcal{D}$-classes since $|\operatorname{im}(\alpha)|=|\operatorname{im}(\beta)|$ for $(\alpha, \beta) \in \mathcal{D}$, and there exist $\left(\begin{array}{l}n \\ p\end{array}\right) \mathcal{R}$-classes and $\left(\begin{array}{l}n \\ p\end{array}\right)$ $\mathcal{L}$-classes in $K_{p}\left(L_{p}\right)$. Moreover, $D P_{n, r}\left(O D P_{n, r}\right)$ is the disjoint union of $K_{0}, K_{1}, \ldots, K_{r}\left(L_{0}, L_{1}, \ldots, L_{r}\right)$.

For $2 \leq p \leq n$ let $\left(d_{1}, \ldots, d_{p-1}\right)$ be a possible gap. Then, let $D_{\left(d_{1}, \ldots, d_{p-1}\right)}$ denotes the $\mathcal{D}$-class which consists of the elements with gap or reverse-gap $\left(d_{1}, \ldots, d_{p-1}\right)$ in $K_{p}$, and similarly denotes the $\mathcal{D}$-class which consists of the elements with gap $\left(d_{1}, \ldots, d_{p-1}\right)$ in $L_{p}$. Notice that all the subsets of $X_{n}$ with the gap $\left(d_{1}, \ldots, d_{p-1}\right)$ are

$$
A_{k}=\left\{k, k+d_{1}, k+d_{1}+d_{2}, \ldots, k+t\right\} \quad \text { for } 1 \leq k \leq n-t
$$


and with the reverse-gap $\left(d_{1}, \ldots, d_{p-1}\right)$ are

$$
B_{k}=\left\{k, k+d_{p-1}, k+d_{p-1}+d_{p-2}, \ldots, k+t\right\} \quad \text { for } 1 \leq k \leq n-t
$$

where $t=\sum_{i=1}^{p-1} d_{i}$. If $\left(d_{1}, \ldots, d_{p-1}\right)$ is symmetric then, since $A_{k}=B_{k}$ for each $1 \leq k \leq n-t$, the $\mathcal{D}$-class $D_{\left(d_{1}, \ldots, d_{p-1}\right)}$ in $K_{p}$ has the following egg box form:

\begin{tabular}{c|c|c|c|}
$D_{s}: R_{1}$ & $\left(\begin{array}{c}A_{1} \\
A_{1}\end{array}\right),\left(\begin{array}{c}A_{1} \\
A_{1}^{R}\end{array}\right)$ & $\cdots$ & $\left(\begin{array}{c}A_{1} \\
A_{n-t}\end{array}\right),\left(\begin{array}{c}A_{1} \\
A_{n-t}^{R}\end{array}\right)$ \\
\cline { 2 - 4 } & & $\ddots$ & \\
\cline { 2 - 4 } & $\left(\begin{array}{c}A_{n-t} \\
A_{1}\end{array}\right),\left(\begin{array}{c}A_{n-t} \\
A_{1}^{R}\end{array}\right)$ & $\cdots$ & $\left(\begin{array}{c}A_{n-t} \\
A_{n-t}\end{array}\right),\left(\begin{array}{c}A_{n-t} \\
A_{n-t}^{R}\end{array}\right)$ \\
\cline { 2 - 4 }
\end{tabular}

If $\left(d_{1}, \ldots, d_{p-1}\right)$ is asymmetric, then the $\mathcal{D}$-class $D_{\left(d_{1}, \ldots, d_{p-1}\right)}$ in $K_{p}$ has the following egg box form:

\begin{tabular}{|c|c|c|c|c|c|c|}
\hline \multirow[b]{2}{*}{$D_{a s}: R_{1}$} & $L_{1}$ & & $L_{n-t}$ & $L_{n-t+1}$ & \multicolumn{2}{|r|}{$L_{2(n-t)}$} \\
\hline & $\left.\begin{array}{l}A_{1} \\
A_{1}\end{array}\right)$ & $\cdots$ & $\left.\begin{array}{c}A_{1} \\
A_{n-t}\end{array}\right)$ & $\begin{array}{c}A_{1} \\
B_{1}^{R}\end{array}$ & $\cdots$ & $\left(\begin{array}{c}A_{1} \\
B_{n-t}^{R}\end{array}\right.$ \\
\hline & & $\ddots$ & & & $\because$ & \\
\hline$R_{n-t}$ & $\begin{array}{c}A_{n-t} \\
A_{1}\end{array}$ & $\ldots$ & $\left.\begin{array}{l}A_{n-t} \\
A_{n-t}\end{array}\right)$ & $\begin{array}{c}A_{n-t} \\
B_{1}^{R} \\
\end{array}$ & $\cdots$ & $\left.\begin{array}{l}A_{n-t} \\
B_{n-t}^{R}\end{array}\right)$ \\
\hline$R_{n-t+1}$ & $\begin{array}{l}B_{1} \\
A_{1}^{R} \\
\end{array}$ & $\cdots$ & $\left.\begin{array}{c}B_{1} \\
A_{n-t}^{R}\end{array}\right)$ & $\left(\begin{array}{l}B_{1} \\
B_{1}\end{array}\right)$ & $\ldots$ & $\left(\begin{array}{c}B_{1} \\
B_{n-t}\end{array}\right)$ \\
\hline & & $\ddots$ & & & $\ddots$ & \\
\hline$R_{2(n-t)}$ & $\begin{array}{c}B_{n-t} \\
A_{1}^{R}\end{array}$ & $\cdots$ & $\begin{array}{l}B_{n-t} \\
A_{n-t}^{R}\end{array}$ & $\begin{array}{c}B_{n-t} \\
B_{1} \\
\end{array}$ & $\cdots$ & $\begin{array}{l}B_{n-t} \\
B_{n-t}\end{array}$ \\
\hline
\end{tabular}

Similarly, the $\mathcal{D}$-class $D_{\left(d_{1}, \ldots, d_{p-1}\right)}$ in $L_{p}$ has the following egg box form:

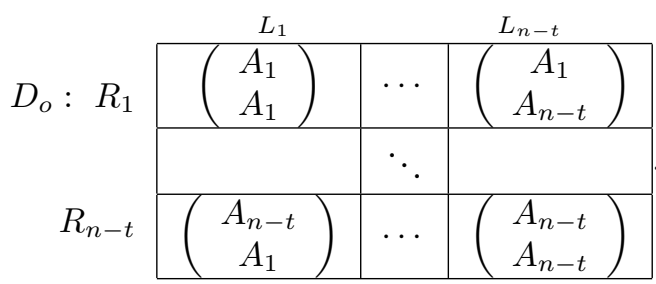

Recall from [2], as a result of [2] Lemmas 1 and 2, that a non-empty subset $W$ of $K_{r}\left(L_{r}\right)$ is a generating set of $D P_{n, r}\left(O D P_{n, r}\right)$ if and only if $K_{r} \subseteq\langle W\rangle\left(L_{r} \subseteq\langle W\rangle\right)$. Moreover, recall that

(I) Let $\alpha_{1}, \ldots, \alpha_{k}$ be some elements of $K_{p}\left(L_{p}\right)$ for $k \geq 2$ and $1 \leq p \leq n-1$. Then $\alpha_{1} \cdots \alpha_{k}=\gamma$ is also an element of $K_{p}\left(L_{p}\right)$ if and only if $\alpha_{i} \alpha_{i+1}$ is element of $K_{p}\left(L_{p}\right)$, equivalently, $\operatorname{im}\left(\alpha_{i}\right)=\operatorname{dom}\left(\alpha_{i+1}\right)$ for each $1 \leq i \leq k-1$.

(II) Let $D$ be a $\mathcal{D}$-class in $D P_{n, r}\left(O D P_{n, r}\right)$ for $2 \leq r \leq n-1$, and let $\alpha_{1}, \ldots, \alpha_{k} \in D$ for $k \geq 2$. Then $\alpha_{1} \cdots \alpha_{k} \in D$ if and only if $\alpha_{i} \alpha_{i+1} \in D$, equivalently, $\operatorname{im}\left(\alpha_{i}\right)=\operatorname{dom}\left(\alpha_{i+1}\right)$ for each $1 \leq i \leq k-1$.

(III) For $2 \leq r \leq n-1$, a non-empty subset $W$ of $K_{r}\left(L_{r}\right)$ is a generating set of $D P_{n, r}\left(O D P_{n, r}\right)$ if and only if $D \subseteq\langle W \cap D\rangle$ for each $\mathcal{D}$-class $D$ in $K_{r}\left(L_{r}\right)$.

As a final of this section we give some definitions about digraphs. Let $\Pi=(V(\Pi), \vec{E}(\Pi))$ be a digraph where $V(\Pi)$ is the set of vertices and $\vec{E}(\Pi) \subseteq V(\Pi) \times V(\Pi)$ is the list of directed edges. For any $u_{1}, \ldots, u_{k} \in V(\Pi)(k \geq 2)$ (they have not to be distinct) if $\left(u_{1}, u_{2}\right),\left(u_{2}, u_{3}\right), \ldots,\left(u_{k-1}, u_{k}\right) \in \vec{E}(\Pi)$, then $u_{1} \rightarrow u_{2} \rightarrow \cdots \rightarrow u_{k}$ is called a walk from $u_{1}$ to $u_{k}$. In particular, for distinct vertices $u_{1}, \ldots, u_{k} \in V(\Pi)$ where $k \geq 1$, the closed walk $u_{1} \rightarrow \cdots \rightarrow u_{k} \rightarrow u_{1}$ is called a cycle, and the cycle consists of a unique vertex is called loop. Also, for any vertices $u, v \in V(\Pi)$ if $u=v$ or there exists a walk from $u$ to $v$ we say $u$ is connected to $v$, and respectively, the vertex $u=v$ or the walk $u \rightarrow \cdots \rightarrow v$ is also called connection from $u$ to $v$. Let $W_{D}$ be a non-empty subset of any $\mathcal{D}$-class $D$ in $K_{r}\left(L_{r}\right)$. Then we define the digraph $\Gamma_{W_{D}}$ as follows: 
- the vertex set of $\Gamma_{W_{D}}$, denoted by $V=V\left(\Gamma_{W_{D}}\right)$, is $W_{D}$; and

- the directed edge set of $\Gamma_{W_{D}}$, denoted by $\vec{E}=\vec{E}\left(\Gamma_{W_{D}}\right)$, is

$$
\vec{E}=\{(\alpha, \beta) \in V \times V: \alpha \beta \in D\}
$$

(For unexplained terms about digraphs, see [5].)

Theorem 2.1. [[2] Theorem 3] Let $D$ be a D-class in $K_{p}$ for $2 \leq p \leq n-1$, and let $\emptyset \neq W_{D} \subseteq D$. Then $D \subseteq\left\langle W_{D}\right\rangle$ if and only if

(i) for each order-preserving map $\gamma \in D \backslash W_{D}$ there exist $\alpha, \beta \in W_{D}$ such that $\operatorname{dom}(\alpha)=\operatorname{dom}(\gamma)$ and $\operatorname{im}(\beta)=\operatorname{im}(\gamma)$, and at least one walk $\rho$, from $\alpha$ to $\beta$ in $\Gamma_{W_{D}}$ such that the number of order-reversing maps in $\rho$ is even, and

(ii) for each order-reversing map $\gamma^{\prime} \in D \backslash W_{D}$ there exist $\alpha^{\prime}, \beta^{\prime} \in W_{D}$ such that $\operatorname{dom}\left(\alpha^{\prime}\right)=\operatorname{dom}\left(\gamma^{\prime}\right)$ and $\operatorname{im}\left(\beta^{\prime}\right)=$ $\operatorname{im}\left(\gamma^{\prime}\right)$, and at least one walk $\rho^{\prime}$, from $\alpha^{\prime}$ to $\beta^{\prime}$ in $\Gamma_{W_{D}}$ such that the number of order-reversing maps in $\rho^{\prime}$ is odd.

Theorem 2.2. [[2] Theorem 4] For $2 \leq r \leq n-1 \operatorname{rank}\left(D P_{n, r}\right)=\left(\begin{array}{l}n \\ r\end{array}\right)$.

Theorem 2.3. [[2] Theorem 5] Let $D$ be a D-class in $L_{p}$ for $2 \leq p \leq n-1$, and let $\emptyset \neq W_{D} \subseteq D$. Then $D \subseteq\left\langle W_{D}\right\rangle$ if and only if, for each $\gamma \in D \backslash W_{D}$ there exist $\alpha, \beta \in W_{D}$ such that $\operatorname{dom}(\alpha)=\operatorname{dom}(\gamma)$ and $\operatorname{im}(\beta)=\operatorname{im}(\gamma)$, and there exists at least one walk from $\alpha$ to $\beta$ in $\Gamma_{W_{D}}$.

Theorem 2.4. [[2] Theorem 6] For $2 \leq r \leq n-1 \operatorname{rank}\left(O D P_{n, r}\right)=\left(\begin{array}{l}n \\ r\end{array}\right)$.

\section{Minimal generating sets of $D P_{n, r}$}

Lemma 3.1. Let $D$ be a $\mathcal{D}$-class in $K_{p}$, for $1 \leq p \leq n-1$, and let $\emptyset \neq W_{D} \subseteq D$. For any possible subset $A$ of $X_{n}$ let $R_{A}$ and $L_{A}$ be the $\mathcal{R}$-class and $\mathcal{L}$-class, which contain id ${ }_{A}$, in $D$, respectively. Moreover, let $H_{A}=R_{A} \cap L_{A}$.

(i) If $R_{A} \cap W_{D} \subseteq H_{A}$, then $R_{A} \cap\left\langle W_{D}\right\rangle \subseteq H_{A}$.

(ii) If $L_{A} \cap W_{D} \subseteq H_{A}$, then $L_{A} \cap\left\langle W_{D}\right\rangle \subseteq H_{A}$.

Proof. Let $D=D_{\left(d_{1}, \ldots, d_{p-1}\right)}$ be a $\mathcal{D}$-class in $K_{p}$, and then notice that

$$
H_{A}= \begin{cases}\left\{\left(\begin{array}{c}
A \\
A
\end{array}\right),\left(\begin{array}{c}
A \\
A^{R}
\end{array}\right)\right\} & \text { if the gap }\left(d_{1}, \ldots, d_{p-1}\right) \text { is symmetric, } \\
\left\{\left(\begin{array}{c}
A \\
A
\end{array}\right)\right\} & \text { if the gap }\left(d_{1}, \ldots, d_{p-1}\right) \text { is asymmetric. }\end{cases}
$$

(i) If $R_{A} \cap W_{D}=\emptyset$ then $R_{A} \cap\left\langle W_{D}\right\rangle=\emptyset$ since $\operatorname{dom}(\beta) \neq A$ for each $\beta \in\left\langle W_{D}\right\rangle$. Now let $\emptyset \neq R_{A} \cap W_{D} \subseteq H_{A}$, and let $\beta \in R_{A} \cap\left\langle W_{D}\right\rangle$. Then there exist $\beta_{1}, \ldots, \beta_{k} \in W_{D}$ such that $\beta=\beta_{1} \cdots \beta_{k}\left(k \in \mathbb{Z}^{+}\right)$. It follows from (II) that $\operatorname{im}\left(\beta_{i}\right)=\operatorname{dom}\left(\beta_{i+1}\right)$ for each $1 \leq i \leq k-1$, and so $\operatorname{dom}(\beta)=\operatorname{dom}\left(\beta_{1}\right)$. Thus $\beta_{1} \in R_{A}$, and so $\beta_{1} \in R_{A} \cap W_{D}$. Then, from the assumption, we have $\beta_{1} \in H_{A}$. Similarly, since $\operatorname{dom}\left(\beta_{i+1}\right)=\operatorname{im}\left(\beta_{i}\right)=A$ for each $1 \leq i \leq k-1$, it follows that $\beta_{1}, \ldots, \beta_{k} \in H_{A}$, and so $\beta \in H_{A}$, as required.

(ii) It can be proved similarly.

Lemma 3.2. Let $D=D_{\left(d_{1}, \ldots, d_{p-1}\right)}$ be a $\mathcal{D}$-class in $K_{p}$ for $2 \leq p \leq n-1$, such that $\left(d_{1}, \ldots, d_{p-1}\right)$ is asymmetric, and let $\emptyset \neq W_{D} \subseteq D$. If $W_{D}$ contains at least one order-reversing map, and if $\Gamma_{W_{D}}$ is a cycle then the number of order-reversing maps in $W_{D}$ is a positive even number.

Proof. Notice that $D$ has the form as $D_{a s}$ given above. Then, with the same notations, the set of all order-reversing maps with gap $\left(d_{1}, \ldots, d_{p-1}\right)$, and the set of all order-reversing maps with reverse-gap $\left(d_{1}, \ldots, d_{p-1}\right)$ are

$$
\begin{aligned}
& U=\left\{\left(\begin{array}{c}
A_{1} \\
B_{1}^{R}
\end{array}\right), \ldots,\left(\begin{array}{c}
A_{1} \\
B_{n-t}^{R}
\end{array}\right), \ldots,\left(\begin{array}{c}
A_{n-t} \\
B_{1}^{R}
\end{array}\right), \ldots,\left(\begin{array}{c}
A_{n-t} \\
B_{n-t}^{R}
\end{array}\right)\right\} \text { and } \\
& V=\left\{\left(\begin{array}{c}
B_{1} \\
A_{1}^{R}
\end{array}\right), \ldots,\left(\begin{array}{c}
B_{1} \\
A_{n-t}^{R}
\end{array}\right), \ldots,\left(\begin{array}{c}
B_{n-t} \\
A_{1}^{R}
\end{array}\right), \ldots,\left(\begin{array}{c}
B_{n-t} \\
A_{n-t}^{R}
\end{array}\right)\right\}
\end{aligned}
$$


where $t=\sum_{i=1}^{p-1} d_{i}$, respectively.

First of all, let $\mu_{1} \rightarrow \cdots \rightarrow \mu_{l}$ be any walk in $\Gamma_{W_{D}}$, for any $l \geq 3$, such that $\mu_{1}, \mu_{l} \in U$ and $\mu_{2}, \ldots, \mu_{l-1} \notin U$. Then it is clear that, since $\operatorname{im}\left(\mu_{i}\right)=\operatorname{dom}\left(\mu_{i+1}\right)$ for each $1 \leq i \leq l-1$, there exists a unique $2 \leq j \leq l-1$ such that $\mu_{j} \in V$. That is, there exists a unique order-reversing map with reverse-gap $\left(d_{1}, \ldots, d_{p-1}\right)$ between two order-reversing maps with gap $\left(d_{1}, \ldots, d_{p-1}\right)$ in $\Gamma_{W_{D}}$. Similarly, there exists a unique order-reversing map with gap $\left(d_{1}, \ldots, d_{p-1}\right)$ between two order-reversing maps with reverse-gap $\left(d_{1}, \ldots, d_{p-1}\right)$ in $\Gamma_{W_{D}}$.

Now, without loss of generality, suppose that $W_{D}=\left\{\lambda_{1}, \ldots, \lambda_{s}\right\}$ for any $s \geq 2$. If $s=2$, since $W_{D}$ contains at least one order-reversing map and $\Gamma_{W_{D}}$ is a cycle, then it is clear that, without loss of generality, $\lambda_{1}$ has a form $\left(\begin{array}{c}A \\ B^{R}\end{array}\right)$ and $\lambda_{2}$ has a form $\left(\begin{array}{c}B \\ A^{R}\end{array}\right)$, as required, where $A \in\left\{A_{1}, \ldots, A_{n-t}\right\}$ and $B \in\left\{B_{1}, \ldots, B_{n-t}\right\}$. Now let $s \geq 3$, and suppose that there exist only $k \geq 1$ order-reversing maps with gap $\left(d_{1}, \ldots, d_{p-1}\right)$ in $W_{D}$, say $\lambda_{i_{1}}, \ldots, \lambda_{i_{k}}$. Then, since $\Gamma_{W_{D}}$ is a cycle, without loss of generality $\Gamma_{W_{D}}$ has the form

$$
\lambda_{i_{1}} \rightarrow \cdots \rightarrow \lambda_{i_{2}} \rightarrow \cdots \rightarrow \lambda_{i_{k}} \rightarrow \cdots \rightarrow \lambda_{i_{1}}
$$

Since there exists a unique order-reversing map with reverse-gap $\left(d_{1}, \ldots, d_{p-1}\right)$ between two order-reversing maps with gap $\left(d_{1}, \ldots, d_{p-1}\right)$ in $\Gamma_{W_{D}}$, also there exist only $k$ order-reversing maps with reverse-gap $\left(d_{1}, \ldots, d_{p-1}\right)$ in $W_{D}$, and so the number of order-reversing maps in $W_{D}$ is $2 k$, as required.

Theorem 3.1. For $2 \leq r \leq n-1$, let $W$ be a non-empty subset of $K_{r}$ with cardinality $\left(\begin{array}{l}n \\ r\end{array}\right)$. Then $W$ is a minimal generating set of $D P_{n, r}$ if and only if the following conditions are satisfied for each $\mathcal{D}$-class $D=D_{\left(d_{1}, \ldots, d_{r-1}\right)}$ in $K_{r}$.

(i) $|R \cap W|=|L \cap W|=1$ for each $\mathcal{R}$-class $R$ and $\mathcal{L}$-class $L$ in $D$.

(ii) - If $\left(d_{1}, \ldots, d_{r-1}\right)$ is symmetric, then the digraph $\Gamma_{W \cap D}$ is a cycle with $n-t$ vertices and the number of orderreversing maps in $W \cap D$ is an odd number.

- If $\left(d_{1}, \ldots, d_{r-1}\right)$ is asymmetric, then the digraph $\Gamma_{W \cap D}$ is a cycle with $2(n-t)$ vertices and the number of order-reversing maps in $W \cap D$ is a positive even number

where $t=\sum_{i=1}^{r-1} d_{i}$.

Proof. $(\Rightarrow)$ Suppose that $\emptyset \neq W \subseteq K_{r}$ is a minimal generating set of $D P_{n, r}$ with cardinality $\left(\begin{array}{l}n \\ r\end{array}\right)$. Then, from (III), $D \subseteq\langle W \cap D\rangle$ for each $\mathcal{D}$-class $D$ in $K_{r}$. Now let $D=D_{\left(d_{1}, \ldots, d_{r-1}\right)}$ be any $\mathcal{D}$-class in $K_{r}$ and let $t=\sum_{i=1}^{r-1} d_{i}$.

(i) The claim is provided from (III), Theorems 2.1 and 2.2.

(ii) Case 1. Suppose that $\left(d_{1}, \ldots, d_{r-1}\right)$ is symmetric. Then $D$ has the form as $D_{s}$ and it is clear that $|W \cap D|=$ $n-t \geq 1$ since the condition $(i)$ is satisfied. If $|W \cap D|=1$ then we have

$$
D: R_{1}\left(\begin{array}{c}
A \\
A
\end{array}\right),\left(\begin{array}{c}
A \\
A^{R}
\end{array}\right)
$$

where $A$ is the unique subset of $X_{n}$ with symmetric gap $\left(d_{1}, \ldots, d_{r-1}\right)$. It is clear that $D \subseteq\langle W \cap D\rangle$ if and only if $W \cap D=\left\{\alpha=\left(\begin{array}{c}A \\ A^{R}\end{array}\right)\right\}$, and so $\Gamma_{W \cap D}$ is a cycle with a unique vertex $\alpha$, which is an order-reversing map, as required.

If $|W \cap D| \geq 2$ then, from the first condition and Lemma 3.1, there is no element in $W \cap D$ which has a form $\left(\begin{array}{c}A \\ A\end{array}\right)$ or $\left(\begin{array}{c}A \\ A^{R}\end{array}\right)$ for any possible non-empty subset $A$ of $X_{n}$. Hence there is no loop in $\Gamma_{W \cap D}$. Now let $\alpha$ and $\beta$ be two distinct elements of $W \cap D$. Then consider any (order-preserving or order-reversing) map $\gamma \in D$ such that $\operatorname{dom}(\gamma)=\operatorname{dom}(\alpha)$ and $\operatorname{im}(\gamma)=\operatorname{im}(\beta)$. Notice that $\alpha$ and $\beta$ are not in the same $\mathcal{R}$-class and not in the same $\mathcal{L}$-class in $D$, from the first condition, and so $\alpha \neq \gamma, \beta \neq \gamma$, and moreover $\gamma \notin W \cap D$. Since $W$ is a generating set of $D P_{n, r}$, from (III), there exist $\lambda_{1}, \ldots, \lambda_{k} \in W \cap D$ such that $\lambda_{1} \cdots \lambda_{k}=\gamma$ for $k \geq 2$. Then, from (II), we have $\operatorname{dom}\left(\lambda_{1}\right)=\operatorname{dom}(\gamma)=\operatorname{dom}(\alpha)$ and $\operatorname{im}\left(\lambda_{k}\right)=\operatorname{im}(\gamma)=\operatorname{im}(\beta)$, and so $\lambda_{1} \mathcal{R} \alpha$ and $\lambda_{k} \mathcal{L} \beta$. From the first condition $\lambda_{1}=\alpha$ and $\lambda_{k}=\beta$, and so there exists a walk from $\alpha$ to $\beta$ in the digraph $\Gamma_{W \cap D}$. Moreover, for any $\alpha \in W \cap D$, there exists a unique $\lambda \in(W \cap D) \backslash\{\alpha\}$ such that $\operatorname{im}(\alpha)=\operatorname{dom}(\lambda)$ and a unique $\mu \in(W \cap D) \backslash\{\alpha\}$ such that $\operatorname{dom}(\alpha)=\operatorname{im}(\mu)$ from the first condition. That is, there exists a unique edge from $\alpha$ and a unique edge to $\alpha$ in $\Gamma_{W \cap D}$. Therefore, $\Gamma_{W \cap D}$ is a cycle with $n-t$ vertices. 
Now let $W \cap D=\left\{\mu_{1}, \ldots, \mu_{n-t}\right\}$ and without loss of generality suppose that the cycle $\Gamma_{W \cap D}$ is $\mu_{1} \rightarrow \cdots \rightarrow$ $\mu_{n-t} \rightarrow \mu_{1}$. Since any product of some order-preserving maps is also an order-preserving map, it is clear that $W \cap D$ must contain at least one order-reversing map. Now consider the map

$$
\delta= \begin{cases}\left(\begin{array}{c}
A \\
B^{R}
\end{array}\right) & \text { if } \mu_{1}=\left(\begin{array}{c}
A \\
B
\end{array}\right), \\
\left(\begin{array}{c}
A \\
B
\end{array}\right) & \text { if } \mu_{1}=\left(\begin{array}{c}
A \\
B^{R}
\end{array}\right)\end{cases}
$$

for two possible different subsets $A$ and $B$ with symmetric gap $\left(d_{1}, \ldots, d_{r-1}\right)$. It is easy to see from (II) that, to generate the map $\delta$ we have to use the walk $\mu_{1} \rightarrow \cdots \rightarrow \mu_{n-t} \rightarrow \mu_{1}$ in $\Gamma_{W \cap D}$, and $\delta$ can be written only as the product $\left(\mu_{1} \cdots \mu_{n-t}\right)^{k} \mu_{1}$ for some $k \geq 1$. If the number of order-reversing maps in $W \cap D$ is even, then $\mu_{1} \cdots \mu_{n-t}$ is the partial identity map with domain set $\operatorname{dom}\left(\mu_{1}\right)$, and so $\left(\mu_{1} \cdots \mu_{n-t}\right)^{k} \mu_{1}=\mu_{1}$ for each $k \geq 1$. Thus we have $\delta \notin\langle W \cap D\rangle$, which is a contradiction, and so the number of order-reversing maps in $W \cap D$ is odd.

Case 2. Suppose that $\left(d_{1}, \ldots, d_{r-1}\right)$ is asymmetric. Then $D$ has the form as $D_{a s}$ and it is clear that $|W \cap D|=$ $2(n-t) \geq 2$ since the condition $(i)$ is satisfied. Similarly we can show that $\Gamma_{W \cap D}$ is a cycle with $2(n-t)$ vertices and $W \cap D$ must contain at least one order-reversing map. Then, from Lemma 3.2, the result is clear.

$(\Leftarrow)$ Suppose that the conditions are satisfied. Now let $D=D_{\left(d_{1}, \ldots, d_{r-1}\right)}$ be any $\mathcal{D}$-class in $K_{r}$ and let $\gamma \in D$. Then, from the first condition, there exist a unique $\alpha \in W \cap D$ and a unique $\beta \in W \cap D$ such that dom $(\gamma)=\operatorname{dom}(\alpha)$ and $\operatorname{im}(\gamma)=\operatorname{im}(\beta)$.

Case 1. Suppose that $\left(d_{1}, \ldots, d_{r-1}\right)$ is symmetric and recall that $|W \cap D|=n-t \geq 1$. From the second condition $\Gamma_{W \cap D}$ is a cycle with $n-t$ vertices and the number of order-reversing maps in $W \cap D$ is odd. Now suppose that $|W \cap D|=1$. Then we similarly have

$D: R_{1}\left(\begin{array}{c}A \\ A\end{array}\right),\left(\begin{array}{c}A \\ A^{R}\end{array}\right)$

and $W \cap D=\left\{\alpha=\beta=\left(\begin{array}{c}A \\ A^{R}\end{array}\right)\right\}$. Notice that $\gamma=\alpha$ or $\gamma=\alpha^{2}$, and so $D \subseteq\langle W \cap D\rangle$, as required.

Next suppose that $|W \cap D|=n-t \geq 2$. If $\gamma \in W \cap D$ then $\gamma=\alpha=\beta$, as required. If $\gamma \notin W \cap D$ and $\alpha=\beta$, then $\operatorname{dom}(\gamma)=\operatorname{dom}(\alpha), \operatorname{im}(\gamma)=\operatorname{im}(\alpha)$ and $\gamma \neq \alpha$, that is $H \backslash\{\alpha\}=\{\gamma\}$ where $H$ is the $\mathcal{H}$-class contains $\alpha$. Then, without loss of generality, suppose that $W \cap D=\left\{\alpha, \lambda_{1}, \ldots, \lambda_{n-t-1}\right\}$ and that $\Gamma_{W \cap D}$ has a form

$$
\alpha \rightarrow \lambda_{1} \rightarrow \cdots \rightarrow \lambda_{n-t-1} \rightarrow \alpha
$$

It is clear that $\alpha \lambda_{1} \cdots \lambda_{n-t-1}$ is an order-reversing map, and so

$$
\gamma=\alpha \lambda_{1} \cdots \lambda_{n-t-1} \alpha \in\langle W \cap D\rangle .
$$

Finally, if $\gamma \notin W \cap D$ and $\alpha \neq \beta$, then, without loss of generality, suppose that $W \cap D=\left\{\alpha, \lambda_{1}, \ldots, \lambda_{k}, \beta, \mu_{1}, \ldots, \mu_{l}\right\}$ for $k, l \geq 0$ (notice that $k+l+2=n-t)$, and that $\Gamma_{W \cap D}$ has a form

$$
\alpha \rightarrow \lambda_{1} \rightarrow \cdots \rightarrow \lambda_{k} \rightarrow \beta \rightarrow \mu_{1} \rightarrow \cdots \rightarrow \mu_{l} \rightarrow \alpha .
$$

If the number of order-reversing maps in $\left\{\alpha, \lambda_{1}, \ldots, \lambda_{k}, \beta\right\}$ is even, then

$$
\gamma= \begin{cases}\alpha \lambda_{1} \cdots \lambda_{k} \beta & \text { if } \alpha \text { is an order-preserving map, } \\ \alpha \lambda_{1} \cdots \lambda_{k} \beta \mu_{1} \cdots \mu_{l} \alpha \lambda_{1} \cdots \lambda_{k} \beta & \text { if } \alpha \text { is an order-reversing map, }\end{cases}
$$

and so $\gamma \in\langle W \cap D\rangle$. If the number of order-reversing maps in $\left\{\alpha, \lambda_{1}, \ldots, \lambda_{k}, \beta\right\}$ is odd, then

$$
\gamma= \begin{cases}\alpha \lambda_{1} \cdots \lambda_{k} \beta \mu_{1} \cdots \mu_{l} \alpha \lambda_{1} \cdots \lambda_{k} \beta & \text { if } \alpha \text { is an order-preserving map, } \\ \alpha \lambda_{1} \cdots \lambda_{k} \beta & \text { if } \alpha \text { is an order-reversing map, }\end{cases}
$$

and so $\gamma \in\langle W \cap D\rangle$. Thus $D \subseteq\langle W \cap D\rangle$, as required.

Case 2. Suppose that $\left(d_{1}, \ldots, d_{r-1}\right)$ is asymmetric, and recall that $|W \cap D|=2(n-t) \geq 2$. If $\gamma \in W \cap D$ then $\gamma=\alpha=\beta$, as required. If $\gamma \notin W \cap D$ then, since each $\mathcal{H}$-class in $D$ consist of a unique element, we have $\alpha \neq \beta$, 
otherwise $\gamma=\alpha \in W \cap D$ which is a contradiction. Since $\Gamma_{W \cap D}$ is a cycle, from the second condition, there exists a unique shortest walk $\rho$ in $\Gamma_{W \cap D}$ from $\alpha$ to $\beta$. Then it is easy to see that $\gamma=\xi \in\langle W \cap D\rangle$ where $\xi$ is the consecutive product of all elements of $\rho$. Thus $D \subseteq\langle W \cap D\rangle$, as required.

Notice that $|W|=\left(\begin{array}{l}n \\ r\end{array}\right)$ from the first condition. Therefore, it follows from (III) and Theorem 2.2 that $W$ is a minimal generating set of $D P_{n, r}$.

Corollary 3.1. Let $W$ is a minimal generating set of $D P_{n, r}$ for $2 \leq r \leq n-1$, and let $D=D_{\left(d_{1}, \ldots, d_{r-1}\right)}$ be a $\mathcal{D}$-class in $K_{r}$.

(i) If $\left(d_{1}, \ldots, d_{r-1}\right)$ is symmetric and $|W \cap D| \geq 2$, then $W \cap D$ does not contain any partial map which has a form $\left(\begin{array}{c}A \\ A\end{array}\right)$ or $\left(\begin{array}{c}A \\ A^{R}\end{array}\right)$ for any possible subset $A$ of $X_{n}$.

(ii) If $\left(d_{1}, \ldots, d_{r-1}\right)$ is symmetric and $|W \cap D|=1$, or if $\left(d_{1}, \ldots, d_{r-1}\right)$ is asymmetric, then $W \cap D$ does not contain any partial identity map.

\section{Minimal generating sets of $O D P_{n, r}$}

Lemma 4.1. Let $D$ be a $\mathcal{D}$-class in $L_{p}$ for $1 \leq p \leq n-1$, and let $\emptyset \neq W_{D} \subseteq D$. For any possible subset $A$ of $X_{n}$ let $R_{A}$ and $L_{A}$ be the $\mathcal{R}$-class and $\mathcal{L}$-class, which contain id ${ }_{A}$, in $D$, respectively. Moreover, let $H_{A}=R_{A} \cap L_{A}$, that is $H_{A}=\left\{\mathrm{id}_{A}\right\}$.

(i) If $R_{A} \cap W_{D} \subseteq H_{A}$, then $R_{A} \cap\left\langle W_{D}\right\rangle \subseteq H_{A}$.

(ii) If $L_{A} \cap W_{D} \subseteq H_{A}$, then $L_{A} \cap\left\langle W_{D}\right\rangle \subseteq H_{A}$.

Proof. The proof is similar to the proof of Lemma 3.1.

Theorem 4.1. For $2 \leq r \leq n-1$, let $W$ be a non-empty subset of $L_{r}$ with cardinality $\left(\begin{array}{l}n \\ r\end{array}\right)$. Then $W$ is a minimal generating set of $O D P_{n, r}$ if and only if

(i) $|R \cap W|=|L \cap W|=1$ for each $\mathcal{R}$-class $R$ and $\mathcal{L}$-class $L$ in $L_{r}$, and

(ii) for each $\mathcal{D}$-class $D$ in $L_{r}$, the digraph $\Gamma_{W \cap D}$ is a cycle.

Proof. The proof is similar to the proof of Theorem 3.1, by using the fact that, $|H|=1$ for each $\mathcal{H}$-class $H$ in $O D P_{n, r}$.

Corollary 4.1. For $2 \leq r \leq n-1$, any minimal generating set of $O D P_{n, r}$ does not contain any partial identity map except partial identities of singleton $\mathcal{D}$-classes in $L_{r}$.

\section{References}

[1] Al-Kharousi, F., Kehinde, R., Umar, A.: On the semigroup of partial isometries of a finite chain. Comm. Algebra. 44, 639-647 (2016).

[2] Bugay, L., Yağcı M., Ayık, H.: The ranks of certain semigroups of partial isometries. Semigroup Forum. 97, 214-222 (2018).

[3] Ganyushkin, O., Mazorchuk, V.: Classical finite transformation semigroups. Springer-Verlag. London (2009).

[4] Howie, J. M.: Fundamentals of semigroup theory. Oxford University Press. New York (1995).

[5] Wilson, R.J., Watkins, J.J.: Graphs, An Introductory Approach, A First Course in Discrete Mathematics. Jon Wiley \& Sons Inc. Toronto (1990). 


\section{Affiliations}

\section{LEYLA BUGAY}

ADDRESS: Department of Mathematics, Çukurova University, Sarıçam, 01330, Adana, Turkey.

E-MAIL: 1tanguler@cu.edu.tr

ORCID ID: 0000-0002-8316-2763

\section{MELEK YAĞCI}

ADDRESS: Department of Mathematics, Çukurova University, Sarıçam, 01330, Adana, Turkey.

E-MAIL: msenol@cu.edu.tr

ORCID ID: 0000-0002-0457-156X 